\title{
Study of Retinal Nerve Fibre Layer Thickness in Diabetics - with and without Diabetic Retinopathy
}

\author{
Pranaykumar Shinde ${ }^{1}$, Madhumita Prasad ${ }^{2}$, Sachin Vishwanath Daigavane ${ }^{3}$, Abhishek G.U. ${ }^{4}$ \\ 1,3,4 Department of Ophthalmology, Jawaharlal Nehru Medical College, Datta Meghe Institute of Medical \\ Sciences (Deemed University), Sawangi (Meghe), Wardha, Maharashtra,India, ${ }^{2}$ Department of \\ Ophthalmology, Sankara Eye Centre, Indore, Madhya Pradesh, India.
}

\section{ABSTRACT}

\section{BACKGROUND}

Diabetes mellitus is a heterogeneous group of diseases, characterized by a state of chronic hyperglycemia, resulting from varied aetiologies. Diabetic retinopathy (DR) is the most common ocular complication of diabetes with $5 \%$ of diabetics, progressing to severe visual loss of $5 / 200$ or less. ${ }^{3}$ Very few studies have been conducted on optical coherence tomography (OCT) changes in diabetic retinopathy in Indian scenario. We wanted to evaluate the association of retinal nerve fibre layer (RNFL) thickness with diabetic retinopathy and assess the possibility of RNFL thickness changes being a precursor to diabetic retinal changes.

\section{METHODS}

A cross sectional study was conducted at the Department of Ophthalmology, Acharya Vinobha Bhave Rural Hospital. A total of 120 patients were enrolled for the study and divided into four groups of equal population as controls / non-diabetics (NDM), diabetics without retinopathy (NDR), diabetics with non-proliferative diabetic retinopathy (NPDR) and diabetics with proliferative retinopathy (PDR). Patients were evaluated for visual acuity, RNFL thickness, intraocular pressure(IOP), cup disc ratio in each case and data was statistically analysed.

\section{RESULTS}

Mean temporal RNFL thickness in PDR group was 73.72 \pm 15.22 and was statistically significant $(P=0.0001)$ than temporal RNFL thickness in controls $(60.41 \pm 7.56)$, NDR $(61.06 \pm 6.51)$ and NPDR (59.01 \pm 5.51$)$. Mean cup-disc disc ratio was $0.26 \pm 0.08$ in controls, was $0.28 \pm 0.11$ in NDR group, $0.32 \pm 0.08$ in NPDR group and $0.36 \pm 0.11$ in PDR group and was statistically significant $(P=0.026)$. However no statistically significant difference was seen in global average RNFL thickness across groups though it was more in NPDR and PDR when compared to NDM group, and was least in NDR group.

\section{CONCLUSIONS}

There was statistically significant increase in temporal RNFL thickness in diabetic group which worsens with the disease. Hence temporal RNFL thickness may be estimated in diabetic patients to predict transformation to diabetic retinopathy and intervene at an early stage.

\section{KEY WORDS}

Diabetes Mellitus, Retinal Nerve Fibre Layer, Proliferative Diabetic Retinopathy, Non Proliferative Diabetic Retinopathy
Corresponding Author: Dr. Abhishek G. U., Department of Ophthalmology, Jawaharlal Nehru Medical College, Datta Meghe Institute of Medical Sciences (Deemed University), Sawangi (Meghe), Wardha, Maharashtra, India. E-mail: abhishekgudvg@gmail.com

DOI: $10.14260 /$ jemds/2021/497

How to Cite This Article:

Shinde P, Prasad M, Daigavane SV, et al. Study of retinal nerve fibre layer thickness in diabetics - with and without diabetic retinopathy. J Evolution Med Dent Sci 2021;10(31):2427-2432, $10.14260 /$ jemds/2021/497 DOI:

Submission 15-01-2021,

Peer Review 01-06-2021,

Acceptance 08-06-2021,

Published 02-08-2021.

Copyright (C) 2021 Pranaykumar Shinde et al. This is an open access article distributed under Creative Commons Attribution License [Attribution 4.0 International (CC $B Y 4.0)]$ 


\section{BACKGROUND}

Diabetes mellitus (DM) is no longer an epidemic that can be ignored, with majority of patients being concentrated in low and middle income countries. Diabetes mellitus is a heterogeneous group of diseases, characterized by a state of chronic hyperglycaemia, resulting from a diversity of aetiologies both environmental and genetic. Currently the number of cases of diabetes worldwide is estimated to be around 425 million. ${ }^{1}$ This number is predicted to reach 649 million by 2045. World Health Organization (WHO) reports indicate that India tops the world with the largest number of diabetic patients ${ }^{2}$ and India ranks $2^{\text {nd }}$ place in top ten countries with highest number of diabetic patients in the $20-79$ year age group (72.9 million). ${ }^{3}$ Diabetic retinopathy (DR) is the most common ocular complication of diabetes with $5 \%$ of diabetics, progressing to severe visual loss of 5 / 200 or less. ${ }^{4}$ The reduced visibility of the RNFL, the increased optic disc pallor and the unchanged size of the neuroretinal rim and parapapillary atrophy suggest that diabetes mellitus may be associated with nonglaucomatous optic nerve atrophy. ${ }^{5}$

The evolution of newer technologies like the Heidelberg Retina Tomograph (HRT - III), Glaucoma diagnostics - Variable Corneal Compensation (GDx VCC) and OCT have made evaluation of the optic nerve head, the peripapillary area, the macula and the RNFL revolutionary. The resolution and reproducibility of these technologies almost give us a near histological evaluation of the tissue or area we study in the retina. The newer findings in evaluating the optic nerve head $(\mathrm{ONH})$ and retina include $\mathrm{ONH}$ and rim parameters, RNFL thickness along 3.2 to $3.4 \mathrm{~mm}$ - diameter circle around the ONH and macular thickness. ${ }^{6}$

The protocol for management of diabetic retinopathy has been well described in literature, and targets the consequence of vascular damage, secondary to diabetic retinopathy. However, there seems to be a difficulty to explain the tissue level changes in diabetic retinopathy. These changes may have been better documented if histological tissue sections were available. However this is near impossible at the moment in the Seeing Eye. Fortunately advancement in technologies have helped us in obtaining optical section biopsies, through noninvasive means like scanning laser polarimetry (SLP) and optical coherence tomography (OCT). It also helps to quantify the direct effects of diabetic retinopathy on the neurons. Technologies have brought out newer perspectives in understanding and management of DR.

Though studies have been conducted on OCT changes in diabetic retinopathy, very few have been done in Indian scenario. This study was intended to highlight the OCT characteristics of the retinal nerve fibre layer in patients with diabetic retinopathy.

\section{Objectives}

- To evaluate the RNFL thickness in diabetic patients with and without Diabetic retinopathy.

- To determine if the incidence of increased intraocular pressure (IOP) and increased cup to disc ratio is higher in diabetes mellitus patients.

\section{METHODS}

This prospective observational study was conducted from June 2020 to November 2020 at the Department of Ophthalmology, Acharya Vinoba Bhave Rural Hospital, Sawangi by an experienced ophthalmologist under standard conditions. All the patients coming to ophthalmology OPD at AVBRH were selected for the study after taking the inclusion and exclusion criteria into consideration.

\section{Inclusion Criteria}

- Patients with diabetes mellitus (Diabetes mellitus was diagnosed on the basis of the diabetes diagnostic criteria of the World Health Organization). ${ }^{7}$

- $\quad$ Age $>40$ years of age.

\section{Exclusion Criteria}

- Recent ocular surgery (1month)

- Patients $<40$ years of age

- Patients with pseudoexfoliation and pigment dispersion syndrome.

- Thyroid dysfunction,

- $\quad$ Long term steroid users

- High myopia

- Media opacities like cataract

- Other causes for secondary glaucoma

- Post glaucoma surgery

- Diabetic retinopathy with tractional retinal detachment and Post retinal surgery patients.

\section{Sample Size}

120 eyes divided into 4 groups

$\mathrm{n}=\frac{\mathrm{Z}_{\alpha / 2^{2}} \mathrm{P}(1-\mathrm{P})}{\mathrm{d}^{2}}$

$\mathrm{Z}_{\alpha / 2}$ is the level of significance at $5 \%$ i.e $95 \%$ confidence interval $=1.96$

$\mathrm{P}=$ Prevalence of Pterygium $=3.57 \%=0.035$

$\mathrm{D}=$ Derived error of margin $=7 \%=0.07$

$\mathrm{n}=\frac{1.96^{2} \times 0.0847 \times(1-0.0847)}{0.05^{2}}$

$=26.47$

$\mathrm{n} \sim 30$

Therefore 30 eyes in each group. Reference - Raman R, Rani PK, Reddi Rachepalle S, et al. Prevalence of diabetic retinopathy in India. Sankara Nethralaya diabetic retinopathy epidemiology and molecular genetics study report 2 . Ophthalmology 2009;116:311-318. $10.1016 \quad$ / j.ophtha.2008.09.010

- Controls (normal patients without diabetes / NDM) - 30 eyes

- Diabetics without retinopathy (NDR group) - 30 eyes

- Non proliferative diabetic retinopathy (NPDR group) - 30 eyes

- $\quad$ Proliferative diabetic retinopathy (PDR group) - 30 eyes 


\section{Data Collection, Tools and Process}

The study was adhered to the tenets of Declaration of Helsinki and it was approved by institutional Ethics committee of DMIMS (DU) [IEC: DMIMS (DU) / IEC / MAY - 2019 / 8743].

After taking informed consent from all the subjects following inclusion and exclusion criteria, a detailed medical history with special mention to $\mathrm{h} / \mathrm{o}$ diabetes mellitus and $\mathrm{h} / \mathrm{o}$ any drug intake was noted. Family h / o diabetes mellitus and glaucoma was also noted. Complete ocular examination, visual acuity recording, IOP recording by applanation tonometry, fundus examination, (cup to disc ratio was measured with micrometer scale attached to eyepiece of slit lamp) and RNFL analysis by OCT was done to each subject. Blood sugars, $\mathrm{HbA} 1 \mathrm{c}$, S. cholesterol and S. creatinine were routinely done for all patients.

\section{Statistical Analysis}

It was done by using descriptive and inferential statistics using one way ANOVA and multiple comparison: Tukey Test and software used in the analysis were SPSS 24.0 version and GraphPad Prism 7.0 version and $\mathrm{P}<0.05$ was considered as level of significance.

\section{RESULTS}

OCT was done in 120 patients with 30 patients in each group. Maximum number of patients belonged to the age group of 50 - 59 yrs. The study comprised of $35 \%$ females (42) and $65 \%$ males (78) Severity of diabetic retinopathy was directly proportional to duration of DM (Table 1).

\begin{tabular}{|ccc|}
\hline Group & $\begin{array}{c}\text { Mean Duration of Diabetes } \\
\text { in Months }\end{array}$ & SD \\
NDR & 73.01 & 31.51 \\
NPDR & 131.11 & 61.71 \\
PDR & 137.51 & 58.12 \\
\hline Table 1. Duration of Diabetes According to Group \\
\hline \multicolumn{2}{|l}{} \\
\hline
\end{tabular}

\section{Distribution of Patients Taking Insulin}

About $75 \%$ of patients in PDR group and $60 \%$ of patients in NPDR group were taking insulin.

\section{Association with Hypertension}

Total of 50 patients ( $41.6 \%)$ had hypertension

NDM: 9 patients (30\%)

NDR: 18 patients $(60 \%)$

NPDR: 25 patients $(83 \%)$

PDR: 26 patients ( $86 \%$ )

\section{Best Corrected Visual Acuity}

As comparison across all groups showed statistical significance, further individual group comparison with each other was done.

\begin{tabular}{|c|c|c|c|c|}
\hline \multicolumn{2}{|c|}{ DR Groups } & \multicolumn{2}{|c|}{ Mean \pm SD } & F - Value \\
\hline \multicolumn{2}{|c|}{ NDM } & \multicolumn{2}{|c|}{$0.79 \pm 0.10$} & \\
\hline \multicolumn{2}{|c|}{ NDR } & \multicolumn{2}{|c|}{$0.81 \pm 0.17$} & \multirow{3}{*}{$\begin{array}{c}19.53 \\
P=0.0001, S\end{array}$} \\
\hline \multirow{2}{*}{\multicolumn{2}{|c|}{$\begin{array}{l}\text { NPDR } \\
\text { PDR }\end{array}$}} & \multicolumn{2}{|c|}{$0.63 \pm 0.12$} & \\
\hline & & \multicolumn{2}{|c|}{$0.43 \pm 0.18$} & \\
\hline \multicolumn{5}{|c|}{ Intra - group comparison of best corrected visual acuity } \\
\hline Group & & reen the & Mean Difference & P - Value \\
\hline \multirow{3}{*}{ NDM } & & & 0.05 & $0.95, \mathrm{NS}$ \\
\hline & & & 0.23 & $0.0001, \mathrm{~S}$ \\
\hline & & & 0.31 & $0.0001, \mathrm{~S}$ \\
\hline \multirow{2}{*}{ NDR } & & & 0.22 & $0.0001, \mathrm{~S}$ \\
\hline & & & 0.37 & $0.0001, \mathrm{~S}$ \\
\hline NPDR & & & 0.13 & $0.047, \mathrm{~S}$ \\
\hline \multicolumn{5}{|c|}{ Table 2. Best Corrected Visual Acuity across Groups } \\
\hline
\end{tabular}

BCVA was significantly worse $(\mathrm{P}<0.005)$ in NPDR when compared with NDM and NDR. Similar was the picture seen on comparing PDR with other groups.

\section{IOP with CCT and Cup Disc Ratio}

There was no statistical significance seen among groups for IOP with CCT but statistical significance was seen for CD ratio across groups, being minimal in NDM group (0.21).

\begin{tabular}{|cccc|}
\hline & DR Groups & Mean \pm SD & F - Value \\
& NDM & $0.21 \pm 0.07$ & \\
IOP with CCT & NDR & $0.26 \pm 0.09$ & 3.22 \\
& NPDR & $0.31 \pm 0.07$ & $\mathrm{P}=0.015, \mathrm{~S}$ \\
& PDR & $0.34 \pm 0.12$ & \\
Cup Disc Ratio across & NDM & $12.63 \pm 2.11$ & \\
Groups & NDR & $13.56 \pm 1.79$ & 0.86 \\
& NPDR & $14.48 \pm 1.98$ & $\mathrm{P}=0.39, \mathrm{NS}$ \\
\hline \multicolumn{4}{c}{ Table 3. IOP with CCT and C:D ratio } \\
\hline
\end{tabular}

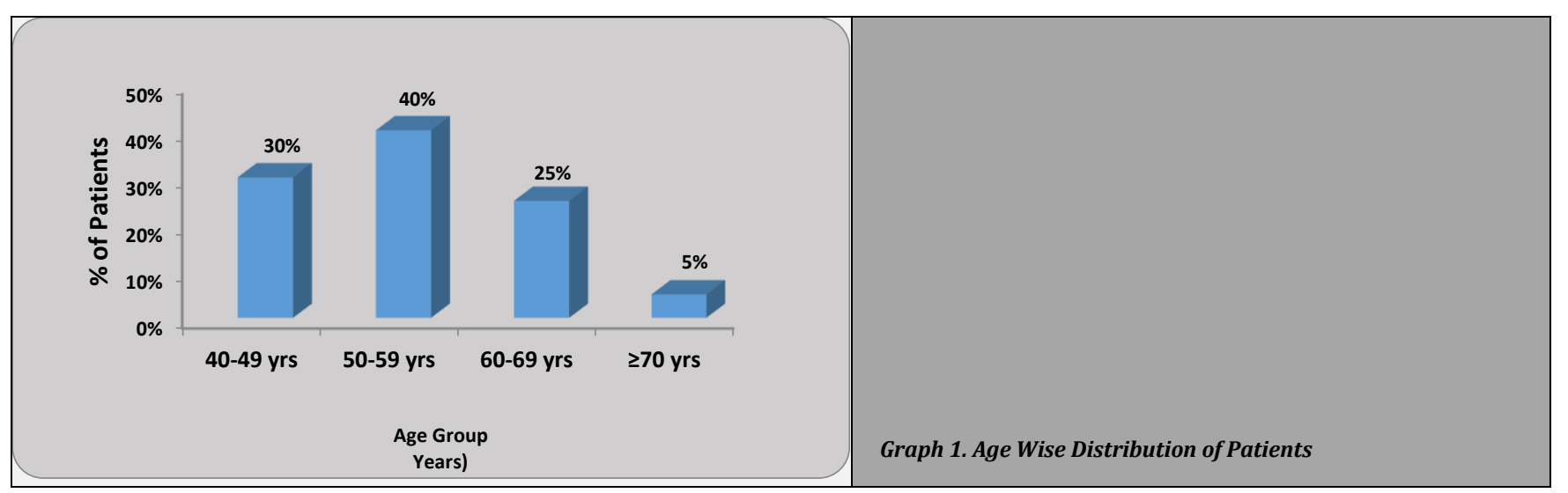




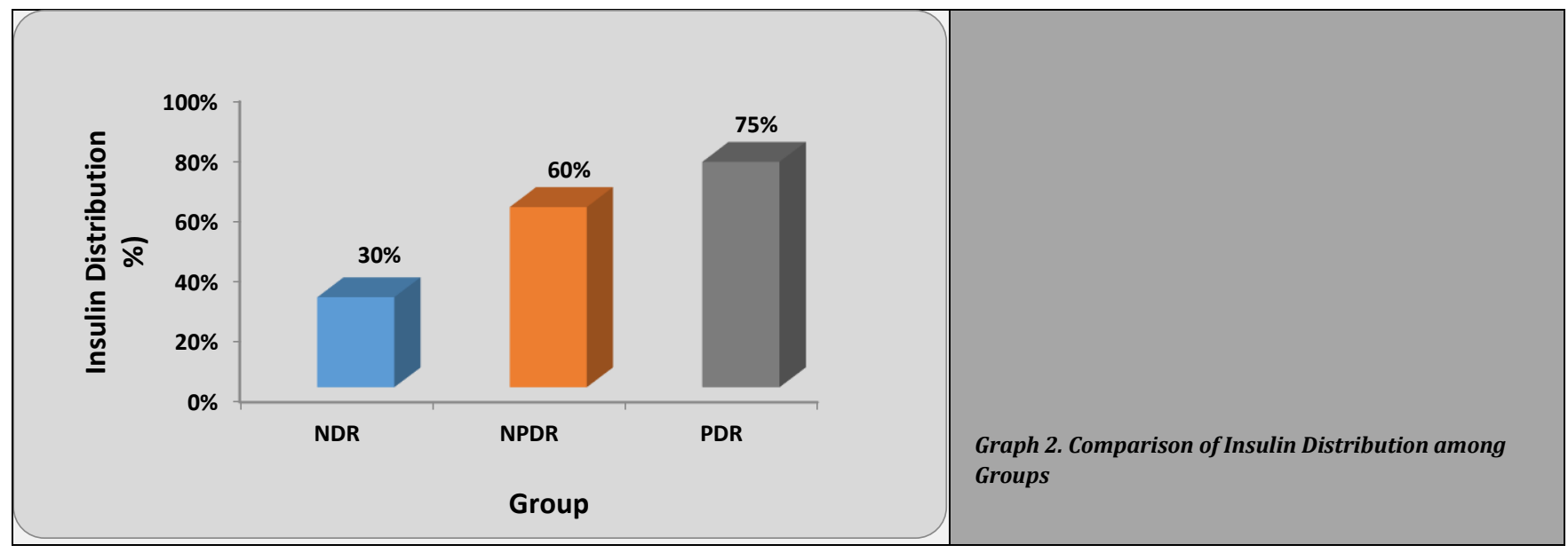

\section{Retinal Nerve Fibre Layer Thickness}

There was no statistical significance seen in average retinal nerve fibre layer $(P=0.101)$, inferior RNFL $(P=0.71)$, superior RNFL $(P=0.28)$ and nasal RNFL $(P=0.19)$. However temporal RNFL thickness comparison was statistically significant.

\section{Temporal Retinal Nerve Fibre Layer Thickness}

\begin{tabular}{|c|c|c|c|}
\hline \multicolumn{4}{|c|}{ Temporal RNFL Thickness across Groups } \\
\hline Groups & Mean \pm SD & \multicolumn{2}{|c|}{ F - Value } \\
\hline NDM & $59.71 \pm 6.51$ & \multirow{4}{*}{\multicolumn{2}{|c|}{$\begin{array}{c}11.53 \\
P=0.0001, S\end{array}$}} \\
\hline NDR & $60.06 \pm 7.21$ & & \\
\hline NPDR & $58.23 \pm 4.31$ & & \\
\hline PDR & $71.72 \pm 13.21$ & & \\
\hline \multicolumn{4}{|c|}{ Between Group Comparison of temporal RNFL Thickness } \\
\hline Group & $\begin{array}{l}\text { Comparison between } \\
\text { the Groups }\end{array}$ & Mean Difference & P - Value \\
\hline \multirow{3}{*}{ NDM } & NDR & 0.61 & $0.96, \mathrm{NS}$ \\
\hline & NPDR & 1.32 & $1.00, \mathrm{NS}$ \\
\hline & PDR & 12.56 & $0.0001, \mathrm{~S}$ \\
\hline \multirow[t]{2}{*}{ NDR } & NPDR & 2.15 & $0.92, \mathrm{NS}$ \\
\hline & PDR & 11.61 & $0.0001, \mathrm{~S}$ \\
\hline NPDR & PDR & 13.73 & $0.0001, \mathrm{~S}$ \\
\hline
\end{tabular}

As there was statistical significance seen across groups for temporal RNFL thickness, comparison was done in between each group. Temporal retinal nerve fibre layer thickness in PDR was significantly more in PDR when compared with NDM, NDR and NPDR groups. However no statistical significance was seen between NDR vs NPDR and NDM vs NPDR groups.

\section{Macular Thickness}

\begin{tabular}{|c|c|c|c|c|}
\hline \multirow{6}{*}{ Macular Thickness across Groups } & Groups & Mean \pm SD & \multicolumn{2}{|c|}{ F - Value } \\
\hline & NDM & $261.41 \pm 10.52$ & \multirow{4}{*}{\multicolumn{2}{|c|}{$\begin{aligned} & 25.23 \\
\mathrm{P}= & 0.0001, \mathrm{~S}\end{aligned}$}} \\
\hline & NDR & $245.65 \pm 26.42$ & & \\
\hline & NPDR & $287.19 \pm 39.51$ & & \\
\hline & PDR & $296.81 \pm 33.21$ & & \\
\hline & Group & $\begin{array}{l}\text { Comparison } \\
\text { between the } \\
\text { Groups }\end{array}$ & $\begin{array}{c}\text { Mean } \\
\text { Difference }\end{array}$ & P - Value \\
\hline \multirow{6}{*}{$\begin{array}{l}\text { Between Groups Comparison of } \\
\text { Macular Thickness }\end{array}$} & \multirow{3}{*}{ NDM } & NDR & 17.2 & $0.031, \mathrm{~S}$ \\
\hline & & NPDR & 19.4 & $0.051, \mathrm{NS}$ \\
\hline & & PDR & 35.2 & $0.0001, \mathrm{~S}$ \\
\hline & \multirow[t]{2}{*}{ NDR } & NPDR & 36.6 & $0.0001, S$ \\
\hline & & PDR & 53.1 & $0.0001, S$ \\
\hline & NPDR & PDR & 16.8 & $0.119, \mathrm{NS}$ \\
\hline \multicolumn{5}{|c|}{ Table 5. Macular Thickness across Groups } \\
\hline
\end{tabular}

Macular thickness when compared across groups showed statistical significance and again comparison was done in between each group. Macular thickness was significantly more in PDR group when compared with NDM and NDR groups. No statistical significance was present between PDR vs NPDR groups and NDM vs NPDR groups.

\section{Blood Investigations}

NDR: HbA1c-7.51 \pm 1.21 , Serum cholesterol $-191.21 \pm 18.52$, Serum Creatinine - $1.20 \pm 0.86$

NPDR: HbA1c-9.36 \pm 1.96 , Serum cholesterol - $189.41 \pm 20.22$ Serum Creatinine - $1.29 \pm 0.85$

PDR: HbA1c-11.51 \pm 5.11 , Serum cholesterol - $178.46 \pm 20.52$, Serum Creatinine - $1.51 \pm 0.88$ HbA1c was significantly higher in PDR group.

Serum cholesterol comparison showed no significant difference across groups.

Comparison between no DM (NDM), DM (without CSME) and DM (with CSME) Groups

\begin{tabular}{|c|c|c|c|}
\hline Groups & Mean \pm SD & \multicolumn{2}{|c|}{ F - value } \\
\hline NDM & $60.41 \pm 5.29$ & \multirow{3}{*}{\multicolumn{2}{|c|}{$\begin{aligned} & 9.21 \\
P= & 0.0001, S\end{aligned}$}} \\
\hline NCSME & $61.36 \pm 9.21$ & & \\
\hline CSME & $69.25 \pm 14.12$ & & \\
\hline \multicolumn{4}{|c|}{ In Between group comparison of temporal RNFL b / n CSME and non - CSME groups } \\
\hline Group & $\begin{array}{l}\text { Comparison between the } \\
\text { groups }\end{array}$ & Mean Difference & $P$ - Value \\
\hline \multirow{2}{*}{ NDM } & NCSME & 1.92 & $0.91, \mathrm{NS}$ \\
\hline & CSME & 9.56 & $0.0001, \mathrm{~S}$ \\
\hline NCSME & CSME & 8.51 & $0.0001, \mathrm{~S}$ \\
\hline \multicolumn{4}{|c|}{ Comparison of macular thickness (CSME and non CSME groups) } \\
\hline Group & $\begin{array}{l}\text { Comparison between the } \\
\text { groups }\end{array}$ & Mean Difference & P - Value \\
\hline \multirow{2}{*}{ NDM } & NCSME & 7.21 & $0.87, \mathrm{NS}$ \\
\hline & CSME & 52.6 & $0.0001, \mathrm{~S}$ \\
\hline NCSME & CSME & 45.92 & $0.0001, \mathrm{~S}$ \\
\hline \multicolumn{4}{|c|}{ Table 6. Comparison of CSME and Non CSME Groups } \\
\hline
\end{tabular}

Temporal RNFL thickness was significantly more in CSME group than Non CSME group. No statistical significance was seen between NDM VS Non CSME groups.

\section{Macular Thickness}

Macular thickness was significantly more in CSME group when compared with non CSME group. However no significant difference was seen between NDM group and NCSME group. 


\section{DISCUSSION}

In our study maximum number of patients belonged to $50-59$ yrs age group. $35 \%$ of total patients were females and $65 \%$ were males. As seen in Table 1, Diabetic retinopathy worsened with increased duration of DM. From table 4 it is evident that with worsening and poor diabetic control diabetic retinopathy also worsens. Study by Raghavendra Ramappa et al. ${ }^{8}$ also concluded with similar trends regarding duration of DM and diabetic control.

\section{BCVA}

Best corrected visual acuity comparison was statistically significant and worse in PDR as compared to NPDR and NDR groups. BCVA was worse in NPDR when compared with NDR group. No significant difference was observed between NDR and NDM groups. This worsening of BCVA in PDR and NPDR may be attributed to occurrence of macular edema in both the groups. In order to confirm, we further classified the patients as NDM, NCSME (no CSME) and CSME groups and analysed the data. BCVA was significantly worse in CSME group when compared with NCSME group and no significant difference was seen between NCSME group and NDM groups hence indicating that worsening of BCVA in DR is due to macular changes. Study by Sánchez - Tocino $\mathrm{H}^{9}$ showed results consistent with our study indicating that worsening of BCVA in diabetics correlated with increase in thickness in central fovea. Study by Ramappa R et al. ${ }^{8}$ showed similar results for BCVA comparison.

\section{IOP with CCT}

There was no statistical significance when IOP was compared across groups. Kay T. Khine ${ }^{10}$ also showed similar results where in the concluded IOP had no significant effect on severity of diabetic retinopathy. Andreas G ${ }^{11}$ also concluded in his study with similar results regarding IOP and severity of DM.

\section{Cup Disc Ratio}

Comparison of cup disc ratio across groups revealed that there was statistical significance across groups with $C D$ ratio being minimal in NDM and maximum in PDR group thus establishing positive corelation between $\mathrm{CD}$ ratio and severity of diabetic retinopathy. Our study results were supported by another study conducted by Pekel E et al. ${ }^{12}$ where in they concluded that $\mathrm{CD}$ ratios were statistically significant and higher in diabetic eyes.

\section{Retinal Nerve Fibre Layer Thickness}

Average, superior, inferior and nasal RNFL thickness in NDM were 93.19 microns, 112.51 microns, 120.12 microns and 70.31 microns respectively and were not statistically significant across groups. Our study did not show any significant difference in terms of average, superior, inferior and nasal retinal nerve fibre layer thickness. Temporal retinal nerve fibre layer was statistically significant and more in PDR when compared with NPDR, NDR and NDM groups. There was no significant difference in temporal RNFL thickness in between NPDR, NDR and NDM groups, thus indicating early onset of neuro retinal changes than vascular changes in increasing severity of DR. Again on further comparison temporal RNFL thickness was significantly more in CSME group as compared with NDM group $(\mathrm{P}=0.0001)$ and Non CSME group.

In a study by Borooah $\mathrm{M}$ et al. ${ }^{13}$ average RNFL thickness in diabetics and non-diabetics was $86.18 \mu \mathrm{m} 91.79 \mu \mathrm{m}$ respectively and was statistically significant. Furthermore among diabetics statistical significance was seen in no DR vs mild DR. RNFL thickness was statistically significant in superior, inferior and temporal quadrants. However in our study only temporal RNFL thickness was statistically significant among groups.

Mohammad AM et al. ${ }^{14}$ in their study showed that there was significant difference in RNFL thickness between NPDR and non-diabetic retinopathy groups. It was noted that in their study superior, temporal and global RNFL thickness was significantly different, RNFL being thicker in NPDR group as compared to non-diabetic retinopathy group.

\section{Macular Thickness}

Macular thickness was highest in PDR group $(296.81 \mu \mathrm{m})$ and was statistically significant when compared with NDM and NDR group. However there was no significant difference seen between NPDR and PDR groups. It was also significantly higher in CSME group when compared with NCSME and NDM groups. No significant difference was seen in NDM and NCSME groups indicating increased RNFL thickness may be due to edema in macular region. Mohammad AM et al. ${ }^{14}$ in their study discussed how macular thickness in NPDR is significantly more in NPDR group when compared to NDR group.

\section{CONCLUSIONS}

Though there was reduction in RNFL thickness in diabetic group it was not clinically significant. However there was statistically significant increase in temporal RNFL thickness in diabetic group which worsened as disease progressed. Hence temporal RNFL thickness may be estimated in diabetic patients to predict transformation to diabetic retinopathy and intervene at an early stage.

Data sharing statement provided by the authors is available with the full text of this article at jemds.com.

Financial or other competing interests: None.

Disclosure forms provided by the authors are available with the full text of this article at jemds.com.

\section{REFERENCES}

[1] https://idf.org/e-library/epidemiologyresearch/diabetes-atlas/134-idf-diabetes-atlas-8thedition.html (accessed on 012/09/2019) (page 7)

[2] Deepa M, Pradeepa R, Rema M, et al. The Chennai Urban Rural Epidemiology Study (CURES)-study design and methodology (urban component) (CURES-1). J Assoc Physicians India 2003;51:863-70. 
[3] https://idf.org/e-library/epidemiologyresearch/diabetes-atlas/134-idf-diabetes-atlas-8thedition.html(accessed on 012/09/2019)(page 46)

[4] Neely KA, Quillen DA, Schachat AP, et al. Diabetic retinopathy. Med Clin North Am 1998;82(4):847-76.

[5] Konigsreuther KA, Jonas JB. Optic disc morphology in diabetes mellitus. Graefes Arch Clin Exp Ophthalmol 1995;233(4):200-4.

[6] Lin SC, Singh K, Jampel HD, et al. Optic nerve head and retinal nerve fiber layer analysis a report by the American academy of ophthalmology. Ophthalmology 2007;114(10):1937-49.

[7] WHO. Definition and diagnosis of diabetes mellitus and intermediate hyperglycemia: report of WHO/IDF consultation. Geneva, Switzerland: World Health Organization 2006.

[8] Ramappa R, Thomas RK. Changes of macular and retinal nerve fibre layer thickness measured by optical coherence tomography in diabetic patients with and without diabetic retinopathy. Int J Sci Stud 2016;3(12):27-33.

[9] Sanchez-Tocino H, Alvarez-Vidal A, Maldonado MJ, et al. Retinal thickness study with optical coherence tomography in patients with diabetes. Invest Ophthalmol Vis Sci 2002;43(5):1588-94.

[10] Khine KT, Guduru A, Challa P, et al. The influence of intraocular pressure on the severity of diabetic retinopathy. Invest Ophthalmol Vis Sci 2017;58(8):66

[11] Bouzas AG, Gragoudas ES, Balodimos MC, et al. Intraocular pressure in diabetes. Relationship to retinopathy and blood glucose level. Arch Ophthalmol 1971;85(4):423-7.

[12] Pekel E, Tufaner G, Kaya H, et al. Assessment of optic disc and ganglion cell layer in diabetes mellitus type 2. Medicine (Baltimore) 2017;96(29):e7556.

[13] Borooah M, Nane YJ, Ekka J. Evaluation of thickness of retinal nerve fiber layer and ganglion cell layer with inner plexiform layer in patients without diabetic retinopathy and mild diabetic retinopathy in type 2 diabetes mellitus patients using spectral-domain optical coherence tomography. Int J Res Med Sci 2018;6(7):2434-9.

[14] El-Hifnawy MAM, Sabry KM, Gomaa AR, et al. Effect of diabetic retinopathy on retinal nerve fiber layer thickness. Delta Journal of Ophthalmology 2016;17(3):162-6. 